\section{Análise do nível de coesão de grupo e do estresse psicológico pré-competitivo de atletas adultos de voleibol}

\section{Analysis of group cohesion levels and pre-competitive psychological stress in volleyball athletes}

Guilherme Moraes Balbim ${ }^{1}$

José Roberto Andrade do Nascimento Junior ${ }^{1}$

Lenamar Fiorese Vieira

Resumo - Este estudo objetivou analisar o nível de coesão de grupo e o estresse psicológico pré-competitivo de atletas adultos de voleibol. Foram sujeitos 155 atletas do gênero masculino e feminino do Estado do Paraná que disputaram a fase final do JAPS 2010/ divisão principal (A) e acesso (B). Como instrumentos, utilizaram-se o Questionário de Ambiente de Grupo (QAG) e o Teste de Estresse Psíquico para o Voleibol (TEP-V). Para análise dos dados, aplicaramse-se o alfa de Cronbach, teste Kolmogorov-Smirnov, Mann-Whitney, Anova One Way e Post Hoc de Tuckey $(\mathrm{p}<0,05)$. Os resultados evidenciaram: os atletas da divisão A foram mais influenciados negativamente pelos fatores de estresse em comparação aos atletas da divisão $\mathrm{B}$; os jogadores da divisão $\mathrm{B}$ apresentaram níveis mais altos de coesão de grupo que os atletas da divisão $\mathrm{A}$, nas dimensões de atração individual para o grupo social $(\mathrm{p}=0,014)$ e tarefa $(\mathrm{p}=0,016)$; os atletas com baixo nível de coesão social foram influenciados negativamente pelos fatores de "Condicionamento físico inadequado" e "Nervosismo excessivo"; atletas com baixo nível de coesão para a tarefa foram influenciados negativamente pelo fator de "Pressão de outras pessoas para ganhar" e positivamente pelo fator "Comportamento da torcida no jogo fora". Concluiu-se: a coesão de grupo demonstra ser um fator interveniente no estresse pré-competitivo, indicando que quanto mais alta a categoria de disputa mais a influência negativa do estresse. Palavras-chave: Atleta; Coesão de grupo; Esporte; Estresse psicológico.

Abstract - This descriptive study was designed to analyze the level of group cohesion and pre-competitive psychological stress of adult volleyball athletes. The subjects consisted of 155 male and female athletes from the state of Parana who played in the JAPS/ $1^{\text {st }}$ division $(A)$, and $2^{\text {nd }}$ division (B). The assessment instruments used were the Group Environment Questionnaire (GEQ) and the Volleyball Psychic Stress Test (V-PST). For data analysis, the following tools were used: Cronbach's alpha, Kolmogorov-Smirnov, Mann-Whitney, one-way Anova, and Tukey's post hoc $(p<0.05)$. The results revealed that athletes of division $A$ were more negatively influenced by stress factors than the athletes of division $B$; in terms of individual attraction to the social group $(p=0.014)$ and tasks $(p=0016)$ athletes of division $B$ had higher levels of group cohesion than athletes from division $A$; athletes with low social cohesion were negatively impacted by factors of "inappropriate physical conditioning" and "excessive nervousness;" athletes with low levels of cohesion for the task were negatively influenced by the factors of "Pressure from other people to win" and positively by the factor "Behavior of the fans in the game outside." It was concluded that group cohesion is demonstrated to be an intervening factor in pre-competitive stress thereby demonstrating that the higher the dispute level, the higher the negative influence of stress.

Key words: Athlete; Group cohesion; Sport; Psychic stress.
1 Universidade Estadual de Maringá. Programa de Pós-Graduação Associado em Educação Física UEM/UEL. Londrina. PR. Brasil

Recebido em: 15/03/12 Revisado em: 30/04/12 Aprovado em: 14/06/12 


\section{INTRODUÇÃO}

Estudos apontam que a coesão de grupo é um fator fundamental para o desempenho de atletas e para sucesso de equipes esportivas ${ }^{1,2}$, visto que é um processo dinâmico que tem como finalidade favorecer a união de um grupo para o alcance de suas metas e satisfação das necessidades afetivas dos membros ${ }^{3}$. No entanto, alguns fatores individuais podem interferir no ambiente e no desempenho de uma equipe ${ }^{4}$, como é o caso do estresse psicológico pré-competitivo que pode ser um elemento interveniente no comportamento e rendimento dos atletas ${ }^{5}$.

Observa-se na literatura que os avanços das pesquisas em relação à coesão de grupo estão relacionados a estratégias de intervenção ao longo da temporada ${ }^{6}$, liderança do treinador ${ }^{7,8}$, eficácia coletiva ${ }^{9}$, desempenho ${ }^{10}$, satisfação de atletas ${ }^{11} \mathrm{e}$ ansiedade ${ }^{12}$. Enquanto que as pesquisas voltadas ao estresse têm se relacionado com diferentes modalidades coletivas e individuais ${ }^{13,14}$, estratégias de coping ${ }^{15,16}$, jovens atletas ${ }^{17}$ e overtraining ${ }^{18}$.

O presente estudo tem como base teórica o modelo conceitual de coesão no esporte de Carron et al. ${ }^{19}$, o qual aponta que a coesão de grupo pode ser influenciada negativa ou positivamente pelos aspectos psicológicos individuais dos atletas, como o estresse psicológico pré-competitivo ${ }^{12}$. Considerando que o estresse pode interferir na coesão de grupo e no desempenho do atleta, evidencia-se a relevância de estudos que abordem estas duas variáveis ${ }^{3,20}$.

Apesar de a literatura apontar a importância da coesão de grupo e do estresse pré-competitivo para o desempenho de uma equipe esportiva e da manutenção de um satisfatório ambiente de grupo para a minimização do estresse pré-competitivo, até onde se sabe não existem estudos no cenário científico da psicologia do esporte que abordem estas duas variáveis em conjunto, sendo esta a lacuna que o presente estudo pretende explorar. Dessa forma, pesquisas neste âmbito podem auxiliar treinadores na preparação psicológica individual e coletiva de suas equipes, com o intuito de otimizar o ambiente da equipe e, consequentemente, o desempenho dos atletas.

Face ao exposto, o presente estudo teve como objetivo analisar o nível de coesão de grupo e o estresse psicológico pré-competitivo dos atletas de voleibol do Estado do Paraná, tendo como hipótese conceitual que atletas com maiores níveis de coesão perceberão menor influência negativa dos fatores estressantes para o desempenho esportivo.

\section{PROCEDIMENTOS METODOLÓGICOS}

\section{Participantes}

Foram convidadas a participar do estudo todas as equipes adultas do gênero masculino e feminino de voleibol classificadas para a fase final dos Jogos Abertos do Paraná (JAPS) 2010, totalizando 240 atletas. No entanto, devido ao não consentimento ou não disponibilidade de algumas equipes, fizeram parte da amostra atletas de 12 equipes das divisões A (principal) 
e B (acesso) participantes do JAPS, sendo 60 do gênero masculino e 95 do gênero feminino, totalizando 155 atletas com mediana de idade de 21 anos.

\section{Instrumentos}

Para avaliar a percepção de coesão de grupo dos atletas, utilizou-se o Questionário de Ambiente de Grupo, adaptado e validado para a língua portuguesa por Borrego et al. ${ }^{21}$, a partir do questionário desenvolvido originalmente por Carron et $\mathrm{al}^{19}$. O instrumento é composto por 13 itens que avaliam a coesão de grupo em equipes esportivas. Os itens são distribuídos em quatro dimensões (Integração no Grupo-Tarefa, Integração no Grupo-Social, Atração Individual para o Grupo-Tarefa e Atração Individual para o Grupo-Social), sendo as afirmações respondidas numa escala tipo Likert de nove pontos que variam de "discordo totalmente" (1) a "concordo totalmente" (9). As pontuações mais altas significam valores mais elevados de coesão em cada uma das dimensões. Para verificar o tipo de coesão predominante das equipes, optou-se por estratificar as quatro dimensões do instrumento em: Coesão Social (média da Integração no Grupo-Social e Atração Individual para o Grupo-Social) e Coesão para a Tarefa (média da Integração no Grupo-Tarefa e Atração Individual para o Grupo-Tarefa). O alfa de Cronbach para todas as dimensões do questionário foram consideradas aceitáveis uma vez que esteve entre $\alpha=0,66$ e $\alpha=0,75$.

Para verificar o nível de estresse psicológico pré-competitivo dos atletas, foi utilizado o Teste de Estresse Psíquico para o Voleibol (TEP-V) que aborda questões sobre as situações e condições que podem representar uma carga psíquica em competições. O instrumento foi elaborado e validado por Noce $^{22}$ e é composto por 30 itens que se referem às situações que podem interferir no rendimento dos atletas. Os participantes deveriam registrar as suas respostas de acordo com o grau de influência negativa ou positiva do estímulo estressor $(-3$ até +3$)$. Os valores do alfa de Cronbach para todos os itens do questionário foram considerados aceitáveis, uma vez que esteve entre $\alpha=0,87$ e $\alpha=0,92$.

\section{Procedimentos}

A pesquisa está integrada ao projeto institucional sob parecer do Comitê de Ética em Pesquisa com Seres Humanos no 175/2007 (Universidade Estadual de Maringá). Inicialmente, foi solicitada a autorização do diretor e da comissão técnica das equipes de voleibol. A coleta de dados foi realizada de forma coletiva, com duração média de 20 a 30 minutos, durante a fase final dos Jogos Abertos do Paraná, no município de Maringá-PR, de acordo com a disponibilidade das equipes e agendamento dos técnicos. Os dados foram coletados no segundo semestre de 2010.

\section{Análise dos dados}

Para a verificação da distribuição dos dados, utilizou-se o teste de Kolmogorov-Smirnov. Como os dados não apresentaram distribuição normal, foram utilizados Mediana (Md) e Quartis (Q1; Q3) para caracterizar os grupos 
e o Teste "U" de Mann-Whitney para a comparação entre as divisões (A e B) e entre a coesão social e para a tarefa. Para a comparação do estresse em relação ao nível de coesão dos atletas (baixo, moderado e alto), após verificar a homogeneidade das variâncias, optou-se pela Anova One Way, seguida do Post Hoc de Tuckey. A significância adotada foi $p<0,05$.

\section{RESULTADOS}

Verificou-se (Tabela 1) diferença significativa entre as divisões A e B nas dimensões "Atração Individual para o Grupo-Tarefa" $(p=0,014)$ e "Atração Individual para o Grupo-Social" ( $\mathrm{p}=0,016$ ), nas quais os atletas da divisão $\mathrm{B}$ apresentaram maior envolvimento individual com a equipe e para $\mathrm{o}$ esporte em relação aos atletas da divisão A.

Tabela 1. Comparação do nível de coesão dos atletas de voleibol das divisões A e B da fase final do JAPS 2010.

\begin{tabular}{|c|c|c|c|}
\hline \multirow[t]{2}{*}{ Dimensão } & $\begin{array}{c}\text { Divisão A } \\
(n=59)\end{array}$ & $\begin{array}{l}\text { Divisão B } \\
(n=96)\end{array}$ & \multirow[t]{2}{*}{$P$} \\
\hline & Md (Q1; Q3) & Md (Q1; Q3) & \\
\hline Integração no Grupo - Tarefa (GI-T) & $5,8(5,0 ; 6,6)$ & $5,8(5,0 ; 6,8)$ & 0,601 \\
\hline Integração no Grupo - Social (GI-S) & $5,5(4,3 ; 6,5)$ & $5,8(4,5 ; 7,0)$ & 0,066 \\
\hline Atração Individual para o Grupo - Tarefa (AI-T) & $5,3(3,0 ; 7,8)$ & $6,9(5,5 ; 7,7)$ & $0,014^{*}$ \\
\hline Atração Individual para o Grupo - Social (AI-S) & $5,2(4,2 ; 7,0)$ & $6,3(4,6 ; 7,6)$ & $0,016^{*}$ \\
\hline
\end{tabular}

*Diferença significativa $p<0,05$.

As dimensões relacionadas à tarefa e ao social foram estratificadas em dois grupos (Coesão para a Tarefa e Coesão Social), possibilitando observar qual o tipo de coesão predomina nos atletas em geral e de acordo com a divisão de disputa das equipes. Verificou-se que não houve diferença significativa $(\mathrm{p}>0,05)$ entre a coesão para a tarefa $(\mathrm{Md}=6,2)$ e a coesão social $(\mathrm{Md}=5,9)$ dos atletas em geral. Ao comparar a Coesão para a tarefa e Coesão social dentro de cada divisão (A e B), não foi encontrada diferença significativa $(\mathrm{p}>0,05)$.

A Tabela 2 apresenta a comparação dos fatores de estresse em função das divisões A e B do JAPS.

Notou-se que houve diferença significativa entre as divisões A e B nos fatores errar no início do jogo, errar no fim do jogo, dormir mal na noite anterior ao jogo, condicionamento físico inadequado, preparação técnico-tática inadequada, falta de preparação psicológica, conflito com o treinador, conflito com companheiros, conflito com familiares, ser prejudicado pelo árbitro, condições de jogo inadequadas, entrar no jogo machucado, machucar-se durante o jogo e nervosismo excessivo $(\mathrm{p}<0,05)$. Os atletas da divisão A perceberam estes fatores de estresse mais negativos para $o$ desempenho esportivo.

Para a comparação do nível de coesão de grupo em função dos fatores de estresse pré-competitivo, optou-se por categorizar os resultados do nível de coesão em: "Baixo" (1,0 a 3,0), "Moderado" (3,1 a 6,0) e "Alto" (6,1 a 9,0), visto que os autores do instrumento ${ }^{19}$ apontam que quanto mais 
próximo a 1,0, mais baixo o nível de coesão, e quanto mais próximo a 9,0, maior o nível de coesão. Para esta análise, foram realizadas comparações intragrupos (divisões A e B). Em relação aos atletas da divisão B, não foi encontrada diferença significativa $(\mathrm{p}>0,05)$ ao comparar os fatores de estresse em função do nível de coesão (social e para a tarefa).

Tabela 2. Comparação dos fatores de estresse que podem influenciar o rendimento dos atletas de voleibol das divisões A e B da fase final do JAPS 2010.

\begin{tabular}{|c|c|c|c|}
\hline \multirow[t]{2}{*}{ Fatores de estresse } & $\begin{array}{c}\text { Divisão } \mathrm{A} \\
(\mathrm{n}=59)\end{array}$ & $\begin{array}{c}\text { Divisão B } \\
(\mathrm{n}=96)\end{array}$ & \multirow[t]{2}{*}{$P$} \\
\hline & Md (Q1; Q3) & Md (Q1; Q3) & \\
\hline Condicionamento físico inadequado & $-2,0(-2,0 ;-1,0)$ & $0,0(-2,0 ; 1,8)$ & $0,006^{*}$ \\
\hline Preparação técnico-tática inadequada & $-2,0(-3,0 ;-1,0)$ & $-1,0(-2,0 ; 1,0)$ & $0,001^{*}$ \\
\hline Falta de preparação psicológica & $-2,0(-3,0 ; 0,0)$ & $0,0(-2,0 ; 1,0)$ & $0,003^{*}$ \\
\hline Conflitos com os companheiros & $-2,0(-2,0 ; 0,0)$ & $0,0(-2,0 ; 1,8)$ & $0,001^{*}$ \\
\hline Entrar no jogo machucado & $-2,0(-3,0 ;-1,0)$ & $0,0(-2,0 ; 2,0)$ & $0,001^{*}$ \\
\hline Machucar-se durante o jogo & $-2,0(-3,0 ;-1,0)$ & $-0,5(-3,0 ; 2,0)$ & $0,001^{*}$ \\
\hline Nervosismo excessivo & $-2,0(-3,0 ; 0,0)$ & $0,0(-2,0 ; 1,0)$ & $0,001^{*}$ \\
\hline Errar jogadas no início do jogo & $-1,0(-2,0 ; 0,0)$ & $0,0(-1,0 ; 0,0)$ & $0,001^{*}$ \\
\hline Errar jogadas no fim do jogo & $-1,0(-2,0 ; 0,0)$ & $0,0(-2,0 ; 2,0)$ & $0,001^{*}$ \\
\hline Dormir mal na noite anterior ao jogo & $-1,0(-2,0 ; 0,0)$ & $0,0(-2,0 ; 1,0)$ & $0,022^{*}$ \\
\hline Pressão de outras pessoas para ganhar & $-1,0(-2,0 ; 0,0)$ & $0,0(-1,0 ; 1,0)$ & 0,153 \\
\hline Cobrança de si mesmo para ganhar & $-1,0(-2,0 ; 1,0)$ & $-0,5(-2,0 ; 1,0)$ & 0,710 \\
\hline Conflitos com o treinador & $-1,0(-2,0 ; 0,0)$ & $0,0(-2,0 ; 1,0)$ & $0,018^{*}$ \\
\hline Conflitos com os familiares & $-1,0(-2,0 ; 0,0)$ & $0,0(-2,0 ; 0,0)$ & $0,007^{*}$ \\
\hline Mau rendimento nos treinamentos & $-1,0(-2,0 ; 0,0)$ & $0,0(-2,0 ; 1,0)$ & 0,107 \\
\hline Críticas do treinador durante o jogo & $-1,0(-1,0 ; 0,0)$ & $0,0(-1,8 ; 1,0)$ & 0,877 \\
\hline Ser prejudicado pelo árbitro & $-1,0(-3,0 ; 0,0)$ & $0,0(-2,0 ; 2,0)$ & $0,001^{*}$ \\
\hline Instalações e condições de jogo inadequadas & $-1,0(-2,0 ; 0,0)$ & $0,0(-2,0 ; 1,8)$ & $0,001^{*}$ \\
\hline Ser o favorito & $0,0(0,0 ; 2,0)$ & $0,0(-0,8 ; 1,0)$ & 0,110 \\
\hline O time adversário é o favorito & $0,0(-1,0 ; 1,0)$ & $0,0(-1,0 ;-0,8)$ & 0,694 \\
\hline Derrotas anteriores & $0,0(-1,0 ; 0,0)$ & $0,0(-1,0 ; 1,0)$ & 0,256 \\
\hline Demora para iniciar o jogo & $0,0(-1,0 ; 0,0)$ & $0,0(-1,0 ; 0,0)$ & 0,222 \\
\hline Bom rendimento inesperado do adversário & $0,0(-1,0 ; 0,0)$ & $0,0(-1,0 ; 1,0)$ & 0,059 \\
\hline Jogar contra um adversário agressivo & $0,0(-1,0 ; 0,0)$ & $0,0(-1,0 ; 0,0)$ & 0,961 \\
\hline Críticas dos companheiros durante o jogo & $0,0(-1,0 ; 0,0)$ & $0,0(-1,0 ; 1,0)$ & 0,530 \\
\hline Ter perdido para o mesmo adversário & $0,0(-1,0 ; 0,0)$ & $0,0(-1,0 ; 1,0)$ & 0,111 \\
\hline Comportamento da torcida no jogo em casa & $0,0(0,0 ; 2,0)$ & $0,0(-2,0 ; 1,0)$ & 0,091 \\
\hline Comportamento da torcida no jogo fora & $0,0(-1,0 ; 1,0)$ & $0,0(-1,0 ; 1,0)$ & 0,939 \\
\hline Comportamento dos jornalistas e repórteres & $0,0(-1,0 ; 0,0)$ & $0,0(0,0 ; 0,0)$ & 0,253 \\
\hline Jogar após o $25^{\circ}$ ponto em desvantagem & $0,0(-1,0 ; 0,0)$ & $0,0(-1,0 ; 1,0)$ & 0,253 \\
\hline
\end{tabular}

*Diferença significativa $p<0,05$.

A Tabela 3 apresenta os fatores de estresse que apresentaram diferença significativa de acordo com o nível de coesão social e para a tarefa dos atletas da divisão $\mathrm{A}$. 
Tabela 3. Fatores de estresse com diferença significativa em relação ao nível de coesão social e para a tarefa dos atletas de voleibol da divisão A da fase final do JAPS 2010.

\begin{tabular}{|c|c|c|c|c|}
\hline \multirow{3}{*}{ Fatores de estresse } & \multicolumn{3}{|c|}{ Coesão social } & \multirow[t]{2}{*}{$\mathrm{p}$} \\
\hline & Baixo & Moderado & Alto & \\
\hline & Md (Q1; Q3) & Md (Q1; Q3) & Md (Q1; Q3) & \\
\hline \multirow[t]{2}{*}{ Errar no início do jogo } & $0,0(-1,5 ; 0,0)$ & $-1,0(-2,0 ; 0,0)^{a}$ & $-2,0(-2,8 ;-1,0)^{\mathrm{a}}$ & $0,020^{*}$ \\
\hline & \multicolumn{3}{|c|}{ Coesão para a tarefa } & \\
\hline Condicionamento físico inadequado & $3,0(-2,0 ; 3,0)^{\mathrm{b}, \mathrm{c}}$ & $-2,0(-2,8 ;-1,0)^{b}$ & $-1,0(-2,0 ;-1,0)^{c}$ & $0,029 *$ \\
\hline Bom rendimento inesperado do adversário & $2,0(-1,0 ; 2,5)^{d, e}$ & $-0,5(-2,0 ; 0,0)^{d}$ & $-0,5(-1,0 ; 0,0)^{\mathrm{e}}$ & $0,024^{*}$ \\
\hline Mau rendimento nos treinamentos & $3,0(-1,0 ; 3,0)^{f, g}$ & $-1,0(-2,0 ; 0,0)^{f}$ & $-1,0(-2,0 ;-0,3)^{g}$ & $0,016^{*}$ \\
\hline
\end{tabular}

*Diferença significativa $p<0,05$ entre: a) moderado e alto; b) baixo e moderado; c) baixo e alto; $d$ ) baixo e moderado; e) baixo e alto; f) baixo e moderado; g) baixo e alto.

Houve diferença significativa, no fator errar no início do jogo $(\mathrm{p}=0,020)$, entre os atletas com moderado nível de coesão social e os atletas de alto nível de coesão social. Observou-se que os atletas com baixo nível de coesão para a tarefa percebem mais positivamente os fatores condicionamento físico inadequado $(\mathrm{p}=0,029)$, bom rendimento inesperado do adversário $(\mathrm{p}=0,024)$ e mau rendimento nos treinamentos $(\mathrm{p}=0,016)$.

\section{DISCUSSÃO}

Observou-se que atletas da divisão A com baixo nível de coesão de grupo (social e tarefa) perceberam os fatores de estresse psicológico pré-competitivo como mais positivo para o desempenho (Tabela 3). Os jogadores da divisão de acesso (B) apresentaram maiores níveis de atração individual para o grupo, tanto voltado para a tarefa como para o social (Tabela 1). Além disso, verificou-se que (Tabela 2) os atletas da divisão principal (A) perceberam mais negativamente fatores de estresse em comparação aos atletas da divisão de acesso (B).

Os atletas da divisão B apresentaram maiores níveis de atração individual para o grupo (tarefa e social), demonstrando indícios de maiores sentimentos relacionados ao envolvimento pessoal com as tarefas do grupo, produtividade, metas e objetivos, assim como sentimentos sobre o envolvimento pessoal, aceitação e interação social com o grupo ${ }^{19}$. Nesse sentido, estudos têm demonstrado que altos níveis de coesão podem ser considerados um preditor da intenção de permanência na equipe e no esporte $^{3,12,23}$, o que pode ser um fator importante para os atletas da divisão de acesso, que visam ser promovidos para a divisão principal.

Quando estratificadas as dimensões em coesão para a tarefa e social, não foi observada diferença significativa, o que vai de encontro à literatura, que aponta que atletas de alto rendimento apresentam maiores níveis de coesão para a tarefa ${ }^{1,11}$, enquanto que atletas jovens apresentam maiores níveis de coesão social ${ }^{24}$.

No entanto, Carron et al. ${ }^{1}$ apontam que independente do tipo de coesão (tarefa ou social), gênero, tipo de esporte ou experiência, se os níveis de coesão forem altos, existe significativa e positiva relação com a performan- 
ce. Contudo, estudos têm apontado que durante as competições de alto rendimento os atletas tendem a destinar maior enfoque nas metas e união social da equipe como um todo ${ }^{11}$, minimizando, por alguns momentos, seus objetivos individuais ${ }^{1}$, achados que vão de encontro com o presente estudo.

Observa-se que os atletas da divisão principal (A) perceberam os fatores estressantes de forma mais negativa em relação aos atletas da divisão de acesso (B), o que pode estar relacionado ao fato destes atletas sofrerem mais de estresse organizacional, ou seja, a relação entre o indivíduo e as demandas do ambiente associado à organização em que está presente ${ }^{5}$. Além disso, o esporte de alto nível é um evento potencialmente provocador de estresse ${ }^{25}$, uma vez que exige do atleta um desempenho ótimo em todos os momentos ${ }^{26}$.

Entre os fatores apontados como mais estressantes estão alguns que não se relacionam diretamente com o esporte propriamente dito, mas que podem ser considerados fontes indiretas de estresse (conflitos com companheiros, treinador e familiares, pressão de outras pessoas para ganhar, dormir mal na noite anterior do jogo), entretanto, Nicholls et al. ${ }^{27}$ assinalam que estas fontes de estresse também podem ter impacto negativo no desempenho dos atletas. Destarte, Fletcher e Wagstaff ${ }^{28}$ confirmam o estresse organizacional, que não está diretamente envolvido com a prática, como um dos principais fatores que interferem no desempenho de alto nível. Dessa forma, é fundamental que atletas de alto nível possuam adequada preparação psicológica, uma vez que, de acordo com Pensgaard e $\mathrm{Duda}^{29}$, quanto melhor a capacidade de um indivíduo de reagir a eventos estressantes, menos ameaçadoras estas situações serão consideradas e menor será a sensação de perigo ou medo frente a situações estressantes.

Ao comparar as condições de estresse em função do nível de coesão social e para a tarefa, observou-se que os atletas da divisão principal (A) que apresentaram baixo nível de coesão para a tarefa perceberam três fatores relacionados à tarefa (condicionamento físico inadequado, bom rendimento inesperado do adversário e mau rendimento nos treinamentos) como positivos para o desempenho esportivo. Esse achado evidencia que atletas sem foco na tarefa não percebem fatores estressantes relacionados às questões físicas e técnicas do esporte como intervenientes no rendimento esportivo ${ }^{3,13}$.

No tocante à coesão social, percebeu-se que atletas com alto nível de coesão social perceberam o fator errar no início do jogo como negativo para o desempenho, evidenciando que, em modalidades coletivas, como o voleibol, erros no início de um jogo podem influenciar a relação entre os atletas e, consequentemente, o desempenho individual e da equipe ${ }^{15}$.

Nosso estudo apresentou como limitação os atletas serem de apenas um estado do Brasil, impossibilitando a comparação com outras realidades do cenário nacional. No entanto, por ter sido uma pesquisa com a maioria dos atletas adultos de voleibol do estado do Paraná, a pesquisa apresentou uma amostra representativa. 


\section{CONCLUSÕES}

A hipótese conceitual do estudo foi rejeitada, evidenciando que o alto nível de coesão de grupo (Tarefa e Social) em equipes de rendimento não é um elemento interveniente para auxiliar na percepção dos atletas em relação a algumas situações de estresse competitivo. Além disso, percebeu-se que o alto nível competitivo é um fator negativo para a percepção do atleta em relação aos agentes estressores que podem influenciar o desempenho esportivo. Sugere-se o desenvolvimento de mais estudos, abordando tais variáveis, sejam realizados com atletas de outras regiões do Brasil e de outras modalidades esportivas, a fim de que resultados mais conclusivos possam ser apontados.

\section{REFERÊNCIAS BIBLIOGRÁFICAS}

1. Carron AV, Bray SR, Eys, MA. Team cohesion and team success in sport. J Sports Sci 2002;20:119-26.

2. Ramzaninezhad R, Keshtan MH. The relationship between coach's leadership styles and team cohesion in Iran football clubs professional league. Braz J Biomotr 2009;3(2):111-20.

3. Carron AV, Hausenblass HA, Eys MA. Group Dynamics in Sport. Morgantown, WV: Fitness Information Technology, 2005.

4. Brandão MRF, Casal HMV, Machado AA, Rebustini F, Agresta M, Ribeiro FA. Futebol, esporte internacional e identidade nacional. Estudo 1: uma comparação entre Brasil \& Japão. Rev Bras Ciênc Mov 2004;12(1):57-62.

5. Lazarus RS. Coping theory and research: Past, present, and future. Psychosomatics 1993; 55:234-47.

6. Senecal J, Loughead TM, Bloom GAA. Season-long team-building intervention: examining the effect of team goal setting on cohesion. J Sport Exerc Psychol 2008; 30:186-99.

7. Ramzaninezhad R, Keshtan MH, Shahamat MD, Kordshooli SS. The relationship between collective efficacy, group cohesion and team performance in professional volleyball teams. Braz J Biomotr 2009;3(1):31-39.

8. Gomes AR, Pereira AP, Pinheiro AR. Liderança, coesão e satisfação em equipas desportivas: um estudo com atletas portugueses de futebol e futsal. Psicol Reflex Crit 2008;21(3):482-91.

9. Toros T. Analysis the differentiation between perceived coaching behaviors, goal orientation, team cohesion, perceived motivational climate and collective efficacy among the young male basketball players before and after the tournament. $\mathrm{Pa}$ mukkale J Sport Sci 2011;2(1):27-39.

10. Beal BJ, Cohen RR, Burke MJ, Mclendon CL. Cohesion and Performance in Groups: A Meta-Analytic Clarification of Construct Relations. J Appl Psychol 2003;88(6):989-1004.

11. Nascimento Junior JRA, Vieira LF, Sousa EA, Vieira JLL. Nível de satisfação do atleta e coesão de grupo em equipes de futsal adulto. Rev Bras Cineantropom Desempenho Hum 2011;13(2):138-44.

12. Eys MA, Loughead TM, Bray SR, Carron AV. Perceptions of cohesion by youth sport participants. Sport Psychologist 2009;23:330-45.

13. Alix-sy D, Scanff CL, Filaire E. Psychological responses in the pre-competition period in elite soccer players. J Sports Sci Med 2008;7:446-54.

14. Merayo EV. Niveles de estrés-recuperacón em deportistas varones de La província de León a través del cuestionario RESTQ-76. Cuad Psic Deporte 2011;11(2):7-24. 
15. Gan Q, Anshel MH, Kim JK. Sources and cognitive appraisals of acute stress as predictors of coping style among male and female chinese athletes. Int J Sport Exerc Psychol 2009;7:68-88.

16. Hoar SD, Crocker PRE, Holt NL, Tamminem, KA. Gender differences in adolescent athletes' coping with interpersonal stressors in sport: more similarities than differences? J Appl Sport Psychol 2010;22:124-39.

17. Harwood C, Knight C. Stress in youth sport: a developmental investigation of tennis parents. Psychol Sport Exerc 2009;10:447-56.

18. Kellmann M. Preventing overtraining in athletes in hight-intensity sports and stress/recovery monitoring. Scand J Med Sci Sports 2010;10(2):95-02.

19. Carron A, Widmeyer W, Brawley L. The Development of Instrument to assess cohesion in sport teams: The Group Environment Questionnaire. J Sport Psychol 1985;7:244-67.

20. Gouvêa FL. Estresse Psíquico no Voleibol Infanto-Juvenil: avaliação de situações de treino. Saúde Rev 2007;9(21):19-26.

21. Borrego CC, Leitão JC, Silva C, Alves J, Palmi J. Análise factorial confirmatória do Group Environment Questionnaire com atletas portugueses. Avaliação Psicológica 2010; 9(3): 359-69.

22. Noce, F, Samulski, DM (2002). Análise do estresse psíquico em atacantes no voleibol de alto nível. Rev Paul Educ Física 16(2): 113-29.

23. Spink KS, Wilson KS, Odnokon P. Examining the relationship between cohesion and return to team in ice hockey players. Psychol Sport Exerc 2010; 11:6-11.

24. Gomes AR, Machado AA. Liderança, coesão e satisfação em equipes de voleibol portuguesas: indicações da investigação e implicações práticas. In: Brandão MRF, Machado AA. O voleibol e a psicologia do esporte (187-218). São Paulo: Editora Atheneu, 2010.

25. Nicholls AR, Holt NL, Polman RCJ, Bloomfield J. Stressors, coping, and coping effectiveness among professional rugby union players. Sport Psychologist 2006; 20(3): 314-29.

26. Dugdale JR, Eklund RC, Gordon S. Expected and unexpected stressors in major international competition: appraisal, coping and performance. Sport Psychologist 2002; 16(1):21-37.

27. Nicholls AR, Hemmings B, Clough PJ. Stress appraisals, emotions, and coping among international adolescent golfers. Scand J Med Sci Sports 2010;20(2):346-55.

28. Fletcher D, Wagstaff CRD. Organizational psychology in elite sport: its emergence, application and future. Psychol Sport Exerc 2010; 10:427-34.

29. Pensgaard AM, Duda JL. Sydney 2000: The interplay between emotions coping and the performance of Olimpic-Level Athletes. Sport Psychologist 2003;17:253-67.
Endereço para correspondência

Universidade Estadual de Maringá, Centro de Ciências da Saúde. Avenida Colombo, 5790, Zona 07 87020-900 - Maringa, PR. Brasil E-mail: guimoraes.ef@gmail.com 